OPEN ACCESS

Edited by:

Steven C. Ricke,

University of Arkansas, United States

Reviewed by:

Jose Manuel Silván Jiménez, Institute of Food Science Research

(CIAL), Spain

Pallavi Singh,

Northern Illinois University,

United States

*Correspondence:

Jingrang Lu

lu.jingrang@epa.gov

Specialty section:

This article was submitted to

Food Microbiology,

a section of the journal

Frontiers in Microbiology

Received: 22 May 2019

Accepted: 19 September 2019

Published: 22 October 2019

Citation:

Lye D, Struewing I, Gruber TM

Oshima K, Villegas EN and $L u J$

(2019) A Gallus gallus Model for Determining Infectivity of Zoonotic

Campylobacter.

Front. Microbiol. 10:2292. doi: 10.3389/fmicb.2019.02292

\section{A Gallus gallus Model for Determining Infectivity of Zoonotic Campylobacter}

\author{
Dennis Lye ${ }^{1}$, Ian Struewing ${ }^{2}$, Theresa M. Gruber ${ }^{1}$, Kevin Oshima ${ }^{1}$, Eric N. Villegas ${ }^{1}$ and \\ Jingrang $L u^{1 *}$
}

${ }^{1}$ Office of Research and Development, USEPA, Cincinnati, OH, United States, ${ }^{2}$ Pegasus Technical Services, Inc., Cincinnati, $\mathrm{OH}$, United States

To better understand public health implications of waterfowl as reservoirs for zoonotic sources of Campylobacter in recreational waters, we developed a Gallus gallus (chick) model of infection to assess the pathogenicity of environmental isolates of Campylobacter. This method involved exposure of 1-day-old chicks through ingestion of water, the natural route of infection. Viable Campylobacter from laboratory-infected animals were monitored by using a modified non-invasive sampling of fresh chick excreta followed by a passive polycarbonate-filter migration culture assay. The method was used to evaluate the infectivities of three laboratory strains of Campylobacter spp. (Campylobacter coli, Campylobacter jejuni, and Campylobacter lari), three clinical isolates of $C$. jejuni, and four environmental Campylobacter spp. isolated from California gulls (Larus californicus). The results revealed that chicks were successfully infected with all laboratory and clinical isolates of Campylobacter spp. through ingestion of Campylobacter-spiked water, with infection rates ranging from $<10$ to $>90 \%$ in a dose-dependent manner. More importantly, exposure of chicks with Campylobacter spp. isolated from Gallus gallus excreta also resulted in successful establishment of infection ( $\leq 90 \%$ ). Each monitored Campylobacter spp. contained $\geq 7.5 \times 10^{4} \mathrm{CFU} \cdot \mathrm{g}^{-1}$ of feces 7 days post-exposure. These results suggest that a $G$. gallus model can be used to assess infectivity of Campylobacter isolates, including gull and human clinical isolates. Use of an avian animal model can be applied to assess the importance of birds, such as the G. gallus, as potential contributors of waterborne-associated outbreaks of campylobacteriosis.

Keywords: Campylobacter, colonization, chick model, infectivity, gull, avian

\section{INTRODUCTION}

Campylobacter spp. bacteria are a major cause of zoonotic human enteric infections commonly transmitted by ingestion of contaminated food or water (Pitkänen, 2013). These bacteria, which are commensal organisms within the gastrointestinal tract of various animals, including birds, have also been isolated from contaminated fresh and marine recreational beach sites (Savill et al., 2001; Stoddard et al., 2005). Recent studies have suggested that Campylobacter spp. present in a 
water source often reflect the source(s) and/or type of fecal pollution at that site. For example, Campylobacter jejuni is most associated with sewage discharges, whereas Campylobacter coli and Campylobacter lari are associated more with agricultural runoff and/or the presence of abundant waterfowl fecal contamination (Pitkänen, 2013). Because Campylobacter spp. require specific fastidious environmental parameters for growth, they are unable to multiply and persist in most surface waters (Obiri-Danso et al., 2001); thus, detection of culturable Campylobacter spp. in surface waters is usually an indication of recent fecal contamination.

Amid increasing public concerns about transmission of enteric disease from waters harboring large bird populations, fecal releases from wild birds have been reported to have a significant role in water quality impairment of recreational waters (Lévesque et al., 2000). Additionally, seagulls and ducks have been reported to be major contributors of Salmonella and Campylobacter bacteria via release and dispersal of their feces in recreational waters (Kapperud and Rosef, 1983; Quessy and Messier, 1992). The extent of colonization and persistence of various Campylobacter spp. within wild birds remains largely undetermined. Ramos et al. (2010) reported a direct relationship between Campylobacter infection of fledgling gull chicks with exposure/consumption diets within human-altered environments (particularly related to garbage and sewage). The colonized gulls showed no adverse health impacts, which could lead to the potential dispersal of Campylobacter over large geographical areas (Bingham-Ramos and Hendrixson, 2008).

Understanding the role of waterfowl as reservoirs for zoonotic Campylobacter spp. in recreational waters has important public health implications. The animal models (piglet, mouse, and rabbit) to assess the infectivity and pathogenicity of different strains of Campylobacter have been used (Field et al., 1981; Babakhani et al., 1993; Hodgson et al., 1998; Stahl and Vallance, 2015; Giallourou et al., 2018; Hartley-Tassell et al., 2018). Considering that Campylobacter spp., especially C. jejuni, have evolved to preferentially colonize the avian gut, the chick model was developed and is the more relevant animal model for investigating bacterial colonization factors (Newell, 2001; Müller et al., 2006; Manes-Lazaro et al., 2017; Sweeney et al., 2017; Salaheen et al., 2018). Although animal models have also been shown to be useful for investigating Campylobacter virulence factors involved during infection, these approaches have inherent limitations, specifically the use of invasive procedures and surgical intervention and/or atypical administration of bacterial inoculums (via oral inoculation or gavage) that do not mimic natural infections with Campylobacter (Ringoir et al., 2007; Stern, 2008). Invasive sampling procedures, such as terminal surgical tissue (Stern, 2008; Clavijo and Flórez, 2017) or cecal swab sampling (Cawthraw et al., 1996; Ringoir et al., 2007), have also been used. Together, these approaches may alter animal behavior and susceptibility to Campylobacter infection and potentially affect accurate assessment of susceptibility to and persistence of Campylobacter infections.

Detection and isolation of Campylobacter spp. from the environment are laborious and often based on enrichment procedures, selective media, and antibiotic resistance. Moreover, many environmental Campylobacter isolates are sensitive to antibiotics, which makes it difficult to use selective culturebased detection (Steele and McDermott, 1984). Nevertheless, an improved method has been reported that uses a passivefiltration plating technique for isolation of Campylobacter spp. from environmental waters and animal samples; the method uses size exclusion filters to select for "smaller" highly motile Campylobacter from most other "larger" less motile bacteria that may be found in environmental samples (Steele and McDermott, 1984; Jokinen et al., 2012). In a subsequent study, the use of polycarbonate filters gave increased recovery of Campylobacter spp. from stool samples when using the same passive-filtration plating procedure (Nielsen et al., 2013). This passive-filtration technique can be valuable for determining the viability of environmental Campylobacter isolates. Thus, this filter method should be a suitable approach for this study.

The aim of this study was twofold: (1) develop a noninvasive chick model of infection that mimics the natural route of infection to assess the infectivity of environmental Campylobacter isolates, and (2) use the passive-filtration plating technique to assess the bacterial burden in animals following infection, including the persistence of various Campylobacter spp. in a natural host. More broadly, since the prevalence and zoonotic potential of Campylobacter spp. found in wild avian species and recreational waters remains poorly understood (Weis et al., 2014, 2016) the techniques described herein will be useful toward developing more accurate risk assessment models of waterfowlderived Campylobacter spp. human infections in a recreational water exposure scenario.

\section{MATERIALS AND METHODS}

\section{Animals}

Specific pathogen-free fertilized chicken layer eggs (Gallus gallus) were obtained from Charles River Laboratories (North Franklin, CT, United States) and were incubated upon receipt $\left(37-38^{\circ} \mathrm{C}\right.$, at $45-55 \%$ relative humidity) for 21 days, with occasional rotation. On day 18 , the eggs were placed on hatching trays at a temperature range of $31-32^{\circ} \mathrm{C}$ and a relative humidity of $60-65 \%$ and allowed to hatch with no additional rotation. Once the chicks hatched, were fully dry, and were able to walk, they were transferred from the hatchery and placed in an individually ventilated cage (IVC) system with the temperature maintained between 32 and $38^{\circ} \mathrm{C}$ throughout the experiment. The chicks were then randomly assigned to one of the three dose groups (A, B, or C; Supplementary Table S1) per Campylobacter isolate (with 17-23 chicks per group, $n=630$ ). There is no "intermixing" between or among the strains with this system. Once randomly assigned to a dose group and placed into the IVC system, they were given the infected water. Both the chamber and cage system were sterilized prior to placing the eggs and chicks. All animal experiments were approved by the US Environmental Protection Agency (USEPA) Animal Facility Oversight of Institutional Animal Care and Use Committee. 


\section{Bacterial Isolates}

Ten Campylobacter species/isolates were analyzed in this study: three laboratory, three clinical, and four environmental isolates. The laboratory isolates were C. jejuni (ATCC 29428), C. coli (ATCC 33559), and C. lari (ATCC 35221) (American Tissue Culture Collection, Manassas, VA, United States). The clinical isolates were cultured specimens taken from human fecal samples of diagnosed C. jejuni infections (kindly provided by a local hospital doctor). The environmental isolates (58BB: C. lari; 63A: C. jejuni; 64BB: C. lari; 70BB: Campylobacter volucre) (Supplementary Table S2) were obtained from California gull (Larus californicus) fecal samples collected from Southern California Hobie Beach as previously described (Lu et al., 2011). All isolates were grown and maintained on 5\% sheep blood agar plates, as described below. Their physiological and biochemical characteristics were tested and summarized in Supplementary Table S3. For long-term storage, frozen $\left(-80^{\circ} \mathrm{C}\right)$ glycerol stocks were made for each clone used in this study, as previously described (Han et al., 1995).

\section{Inoculum Preparation of Campylobacter}

spp.

Campylobacter spp. were cultured by using sheep blood agar plates (SBAP) containing 5\% sheep blood (VWR International Inc., Radnor, PA, United States) incubated for $48-72 \mathrm{~h}$ at $37^{\circ} \mathrm{C}$, which is considered as optimal growth temperature (Hsieh et al., 2018), in microaerophilic chambers (Mitsubishi AnaeroPack System, Fisher Scientific, Hanover Park, IL, United States). Single Campylobacter colonies were transferred by swab onto four fresh SBAPs and incubated for $48 \mathrm{~h}$ at $37^{\circ} \mathrm{C}$ in a microaerophilic chamber until a full bacterial lawn on each plate was achieved. Lawns from these plates were then harvested by flooding each plate with $20.0 \mathrm{~mL}$ of sterile water and then pooled and diluted until an optical density $640=0.14$ was achieved. This initial suspension of Campylobacter was then diluted 10-fold with sterile water, which typically resulted in titers of about $1 \times 10^{7} \mathrm{CFU}$ $\mathrm{mL}^{-1}$. To make sure that animals would show some infection (colonization) under the lowest dose, two additional 10-fold serial dilutions were prepared in sterile water to give a total of three different inoculum doses of culturable Campylobacter used throughout the study (Supplementary Table S1). The volumes prepared for each inoculum dose were sufficient to provide $100 \mathrm{~mL}$ for each chick for $24 \mathrm{~h}$ use. Since Campylobacter spp. have been shown to be sensitive to light and temperature (Obiri-Danso et al., 2001) and can become non-culturable within $30 \mathrm{~min}$ of exposure to artificial light according to our test (data not shown), fresh Campylobacter suspensions were made for each experiment and protected from light in $15-\mathrm{mL}$ conical tubes wrapped with aluminum foil and kept on ice (or refrigerated) until used.

\section{Infection Through Natural Ingestion of Campylobacter-Containing Drinking Water}

Fifty milliliters of freshly prepared Campylobacter suspensions, as described above, were dispensed into sterile isolator containers (Bio Serv, Flemington, NJ, United States) and placed into individual chick cages. An initial $50-\mathrm{mL}$ inoculum solution was placed in the cages for $8 \mathrm{~h}$ and then replaced with a new $50-\mathrm{mL}$ inoculum solution of the same suspension dilution for an additional $16 \mathrm{~h}$ before replacing with sterile drinking water. The viability of inoculum after $8 \mathrm{~h}$ in the cage only had slight decrease. The total volume of inoculum solution ingested by each chick was monitored and calculated for the entire 24-h exposure period. Total bacterial numbers ingested were calculated on the basis of the volume ingested and densities of Campylobacter suspension (Supplementary Table S1). Uninfected control chicks $(n=22)$ just received the same sterile water and were monitored in a manner similar to that for the infected group. Six hundred and thirty 1-day-old chicks were exposed to three different concentrations of laboratory, gull, and human clinical isolates of Campylobacter spp. and monitored over a 7-day period.

\section{Assessing Bacterial Burden in Chick Feces by Using the Passive-Filtration Culture Method}

Fresh fecal materials collected at two time points ( 2 and 7 days) were assayed by using a modified passive-filtration culture method (Steele and McDermott, 1984; Jokinen et al., 2012). Briefly, individual chicks were placed in a cage containing sterile paper bottoms and allowed to defecate. Within 10-15 min, most chicks had defecated onto the sterile paper, and samples were taken by swiping a sterile swab through the freshly deposited feces. Each fecal swab sample with an average of $390 \mathrm{mg}$ was placed into a tube containing $3.0 \mathrm{~mL}$ of sterile water (held on ice) and agitated to dislodge and disperse the fecal matter. After agitation, $0.1 \mathrm{~mL}$, which contained an average of $13 \mathrm{mg}$ of the fecal specimens, was plated directly onto a SBAP plate (100 $\mathrm{mm}$ in diameter) with two $47-\mathrm{mm}$ diameter polycarbonate filters of $0.6-\mu \mathrm{m}$ pore size (GE Water \& Process Technologies, Addison, IL, United States) placed side by side and on top. The filter pore and diameter size provided optimal results for culturing Campylobacter spp. for this study (data not shown). Samples were then spread gently and evenly across the two filters. Finally, the SBAPs were incubated at $37^{\circ} \mathrm{C}$ for $45 \mathrm{~min}$ (no microaerophilic chamber). After $45 \mathrm{~min}$, the filters were removed from the surface by using a sterile forceps, and the SBAPs (without filters) were placed in a microaerophilic chamber and incubated at $37^{\circ} \mathrm{C}$. Campylobacter colonies were enumerated after $48 \mathrm{~h}$ of incubation. On the basis of the processed 14-27 replicates with no-dilution, the Most Probable Number (MPN) calculation program (version 5) (Jarvis et al., 2010) was used to perform MPN analyses. A number of colonies were randomly tested and confirmed for the targeted strains using qPCR methods (Supplementary Table S4) mentioned in previous study (Lu et al., 2013).

\section{Data Analysis}

To check for normality of the data, the Shapiro-Wilk test was performed. As the data were not normally distributed, the non-parametric Kruskal-Wallis One Way Analysis of Variance on Ranks was used to determine if the differences in the 
median values among treatment groups were greater than would be expected by chance. If a statistically significant difference ( $p \leq 0.05$ ) was found, Dunn's test of multiple comparisons was conducted to isolate the group or groups that differed from the others. All statistical tests among different strains, different doses, were done via SigmaPlot 13.0.

\section{RESULTS}

\section{Comparison Using Spread Plate vs. Passive-Filter Culture Assays of Campylobacter spp. Recoveries}

We compared the traditional spread plate with the passive-filter technique for culturing of three laboratory strains (C. jejuni, C. coli, and C. lari), three clinical strains (C1-3), and four gull isolated strains of Campylobacter species (Table 1). The results revealed differences in total colony counts between the spread plate vs. passive-filter culture techniques. Total colony counts in cultures grown by using the spread plating were higher

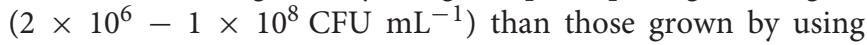

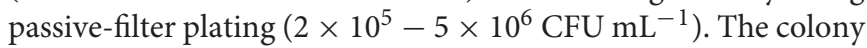
ratio detected between the two plating types ranged from 6 to 30 (mean $=16)$. In addition, the passive-filter technique proved to be less labor intensive and did not require additional sample dilution to avoid overcrowding of the plate (data not shown). On the basis of the added technical advantages and a built-in enrichment step provided by the passive-filter culture technique in this study, as well as on reports by Nielsen et al. (2013), all subsequent experiments described below used this bacterial culture procedure.

TABLE 1 | Comparisons of spread plating vs. passive filter platings to assess total viability of various laboratory, clinical, and environmental Campylobacter strains $\left(\mathrm{CFU} \mathrm{mL}^{-1}\right.$ ).

\begin{tabular}{cccc}
\hline Isolate & $\begin{array}{c}\text { Titer from spread } \\
\text { plating }^{a}\end{array}$ & $\begin{array}{c}\text { Titer from passive } \\
\text {-filter plating }\end{array}$ & $\begin{array}{c}\text { Ratio of spread count } \\
\text { vs. filter count cells }\end{array}$
\end{tabular}

\begin{tabular}{lllc}
\hline Laboratory & & & \\
C. coli & $3 \times 10^{7}$ & $1 \times 10^{6}$ & $30: 1$ \\
C. jejuni & $4 \times 10^{7}$ & $5 \times 10^{6}$ & $8: 1$ \\
C. lari & $1 \times 10^{7}$ & $1 \times 10^{6}$ & $10: 1$ \\
Environmental & & & \\
64BB & $3 \times 10^{7}$ & $2 \times 10^{6}$ & $15: 1$ \\
58BB & $8 \times 10^{6}$ & $6 \times 10^{5}$ & $14: 1$ \\
70BB & $3 \times 10^{7}$ & $5 \times 10^{6}$ & $6: 1$ \\
63A & $5 \times 10^{7}$ & $2 \times 10^{6}$ & $25: 1$ \\
Clinical & & & $10: 1$ \\
C1 & $2 \times 10^{6}$ & $2 \times 10^{5}$ & $20: 1$ \\
C2 & $1 \times 10^{8}$ & $5 \times 10^{6}$ & $25: 1$ \\
C3 & $1 \times 10^{7}$ & $4 \times 10^{5}$ &
\end{tabular}

aTiters calculated from $0.1 \mathrm{~mL}$ of bacterial suspension dilutions plated directly onto BAP plates; 'b Titers calculated from $0.1 \mathrm{~mL}$ of the same bacterial suspension dilutions plated onto BAP-containing polycarbonate filters as described in section "Materials and Methods"; "Ratio of culturable cells calculated from spread plates vs. passive-filter plates.

\section{Infection of Chicks With Campylobacter Laboratory Isolates}

The ability of three laboratory isolates of C. coli, C. jejuni, and C. lari to successfully infect 1-day-old chicks was evaluated. As shown in Figure 1, chicks exposed to various concentrations of Campylobacter spp. (Supplementary Table S1) via a natural ingestion route, as described above, were successfully infected. By contrast, uninfected control animals remained negative for Campylobacter. The three different laboratory strains showed significantly different patterns of infection rates $(p<0.05$, compared at 3-doses and at 2-day time points), which were detected up to 7 days post-infection (Figure 1). C. jejuni had the most consistent colonization rates ranging from 71.4 to $85.7 \%$ of animals that were positive at both time points evaluated and at all three doses. C. lari had the highest infection rates at all three doses at 2 days post-infection (87.5-93.3\%) but had slightly lower infection rates than those of $C$. jejuni by 7 days post-infection (66.7-75.0 vs. $72.2-85.7 \%$, respectively). By contrast, C. coli had the lowest infection rates among the three strains.

When fecal bacterial burden following infection was examined by using MPN, the chicks infected with $C$. lari had the highest detectable bacterial load followed by those infected by $C$. jejuni and C. coli for each dose at 2 days post-infection (Figure 2). Both C. jejuni and C. lari infections were more persistent than those of C. coli. We found that $C$. jejuni easily infected 1-dayold chicks even at the lowest inoculum dose and had the most consistent rate and persistence of infection among the laboratory strains. When inoculated with the same dose as C. jejuni, we found that C. coli infected fewer chicks at the same inoculum dosages (Supplementary Table S1). No overt disease symptoms were observed in the chicks infected by the three Campylobacter species. The natural ingestion exposure route developed in our study enabled successful infection of the young chicks with both the laboratory and clinical strains of Campylobacter (C. jejuni, C. lari, and C. coli) with minimal physiological distress.

\section{Infection of Chicks With Campylobacter Clinical Isolates}

One-day-old chicks were inoculated with three $C$. jejuni isolates collected from patients with campylobacteriosis to determine if human Campylobacter isolates are also capable of establishing infection in this chick model of infection. All three isolates successfully established infection and persisted to 7 days postinfection (Figure 3). Although $\mathrm{C} 1$ showed a consistent infection rate $(81.8 \%)$ at both time points, $\mathrm{C} 2$ and $\mathrm{C} 3$ were more variable. C2 had a lower infection rate at 2 days post-infection $(54.4 \%$, $p \leq 0.019)$ than at 7 days post-infection $(72.7 \%)$, whereas C3 had the reverse trend (91.7 and 25.0\%, respectively).

\section{Infection of Environmental Campylobacter spp. Isolates}

Four California gull isolates (58BB, 63A, 64BB, and 70BB), which were homologous to C. lari ( $\geq 94 \%$ homology), C. jejuni ( $\geq 97 \%$ homology), C. lari ( $\geq 97 \%$ homology), and C. volucri $(\geq 97 \%$ homology), respectively, were capable of infecting chicks via this chick model. C. volucri, a new Campylobacter species isolated 


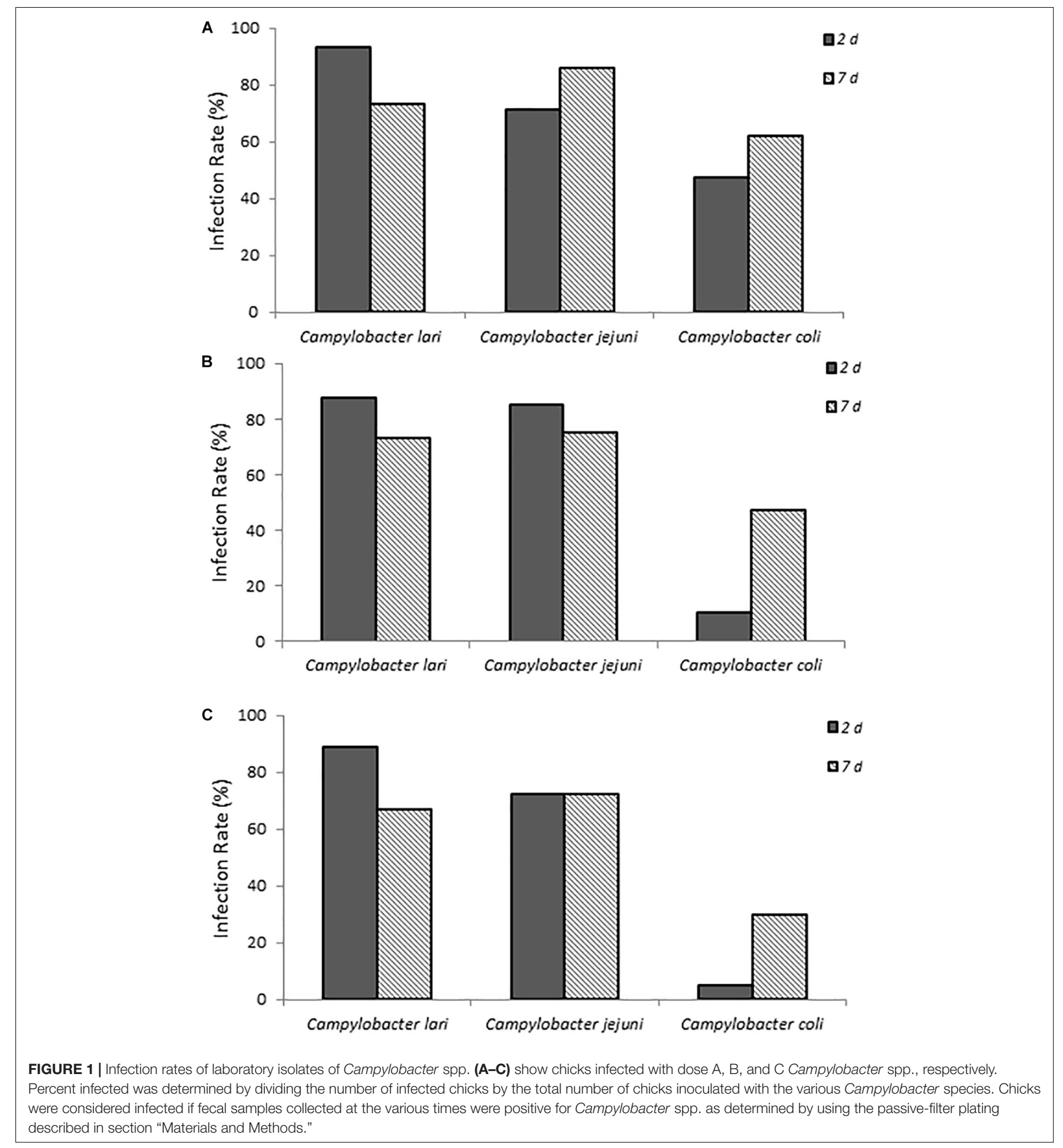

from black-headed gulls (Larus ridibundus) (Debruyne et al., 2010) is more closely related to C. jejuni than to C. lari according to phylogenetic analysis of 16S rRNA and hsp60 gene sequences (Debruyne et al., 2010).

The two C. lari-like isolates (58BB and 64BB) showed the highest infection rates at the two highest doses, with $>80 \%$ of the animals infected at 2 days post-infection and the infection persisting to 7 days post-infection (Figure 4). Even at the lowest dose, $64 \mathrm{BB}$ continued this trend, with $>80 \%$ of the animals infected on both days. At the lowest dose, $58 \mathrm{BB}$ was less infective and infected $<32 \%$ of the animals on each day. By contrast, chicks inoculated with 70BB C. volucri-like or 63A C. jejuni-like isolates had consistently lower rates of infection than those of both $C$. lari-like isolates, with more variations in their infection 


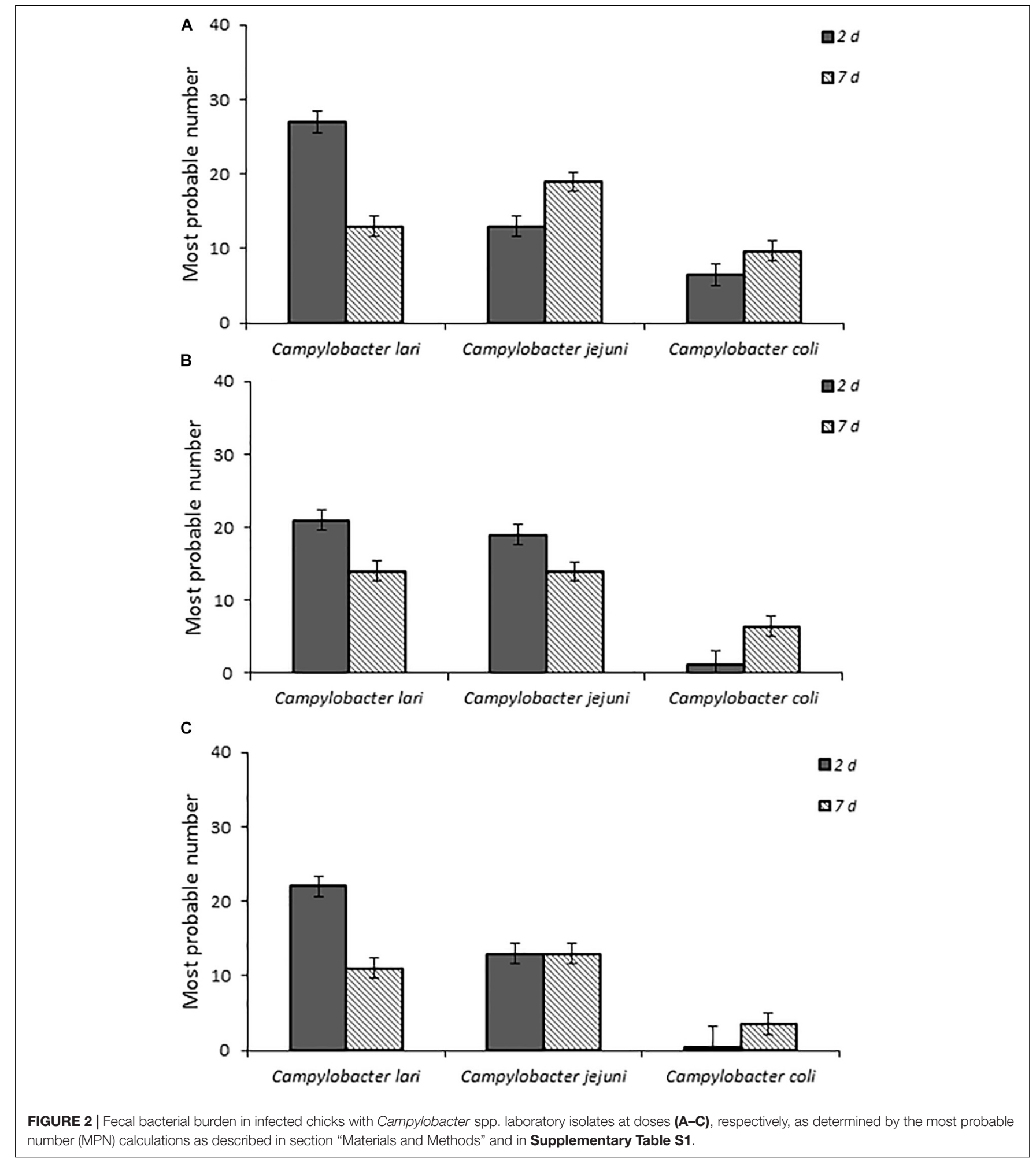

rates. The C. volucri-like isolate had a high infection rate of $72.7 \%$ on 2 days post-infection for dose B, which dropped to $13.6 \%$ by 7 days post-infection. Similarly, the C. jejuni-like isolate had a high infection rate of $70.0 \%$ at 2 days post-infection for dose A, which dropped to $15.0 \%$ at 7 days post-infection (Figure 4).
Chicks that received the highest inoculum dose of $64 \mathrm{BB}$ and $58 \mathrm{BB}$ also had the highest Campylobacter burden, as indicated by MPN, compared with those of $63 \mathrm{~A}$ and $70 \mathrm{BB}$ at both time points tested (Figure 5). The overall Campylobacter burden observed from infection with $63 \mathrm{~A}$ and $70 \mathrm{BB}$, although similar to each other, 

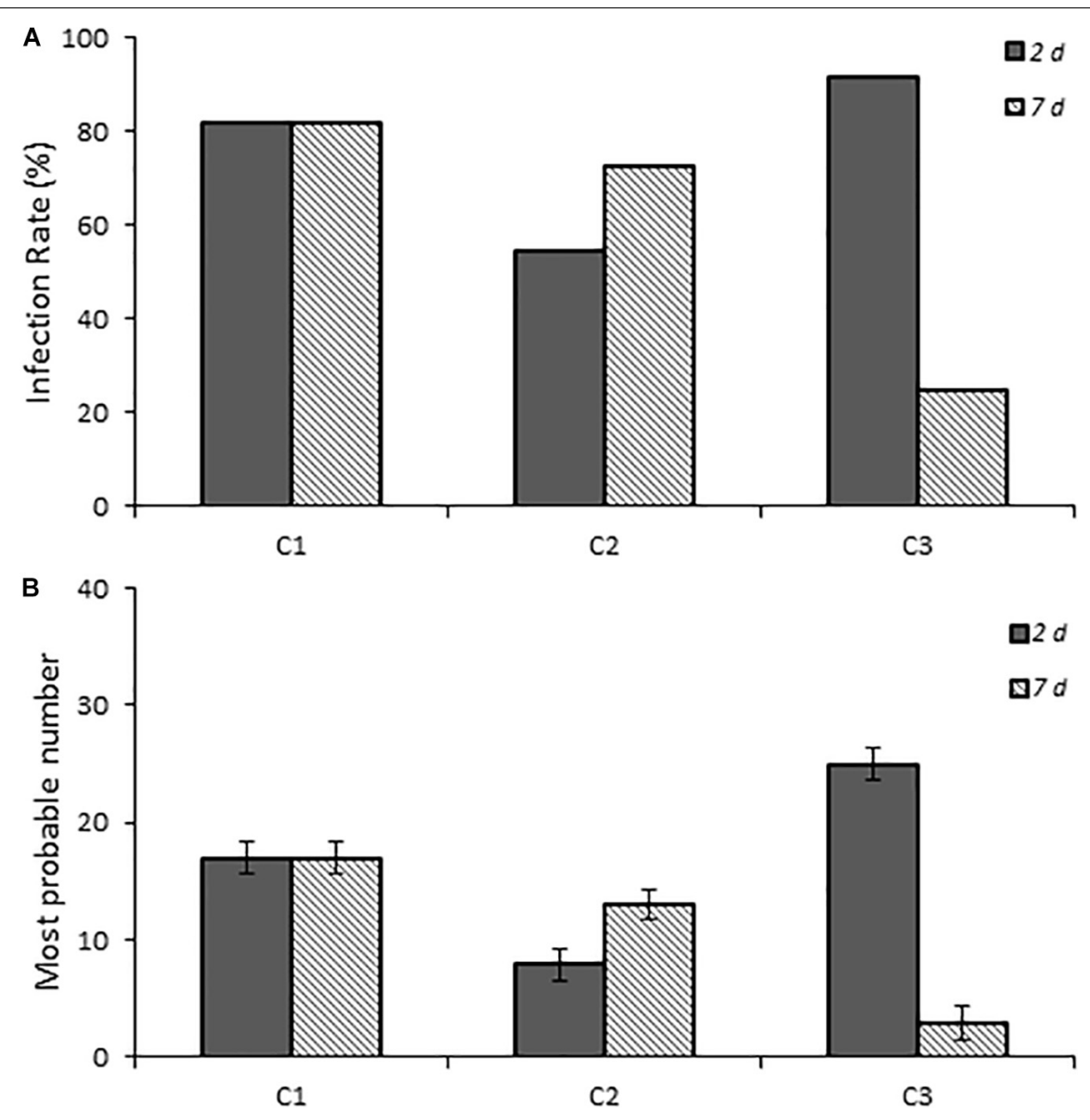

FIGURE 3 | Infection rates and fecal burden levels of Campylobacter from chicks infected with clinical isolates of Campylobacter spp. (A) shows chicks infected with dose B Campylobacter spp. Percent infected was determined by dividing the number of infected chicks by the total number of chicks inoculated with the various Campylobacter species. Chicks were considered infected if fecal samples collected at the various times were positive for Campylobacter spp. as determined by using the passive-filter plating as described in the section "Materials and Methods." (B) shows the fecal burden as measured by using the most probable number calculator.

was lower than the bacterial fecal burdens from animals infected with the $64 \mathrm{BB}$ and $58 \mathrm{BB}$ isolates $(p \leq 0.009)$.

\section{DISCUSSION}

The G. gallus chick species has long been used as an important model for investigation of bacterial colonization factors, especially for Campylobacter infection, because of their preferential colonization of avian guts under optimal growth conditions (Wassenaar et al., 1993; Cawthraw et al., 1996; Hendrixson and DiRita, 2004; Stern, 2008). In the previous studies, bacterial strains, including laboratory-adapted C. jejuni isolates and C. jejuni isolates from chicken, patients, and waterborne outbreaks, were administered via oral gavage to chickens of various ages, tissue or cecal samples were collected, and samples were enumerated by plating of serial dilutions of samples. In those experiments, the cecum was found to be the main site of colonization, although organisms were also recovered throughout the gastrointestinal tract as well as the spleen and liver. Unlike the previous chick models of infection, the model in our studies was one we developed to be less invasive than others by infecting animals with Campylobacter through their drinking water, which mimics the natural route of infection, uses a sampling procedure that measures bacterial burden from freshly excreted fecal samples, and uses a passive filtration in vitro culture procedure as a more feasible model to assess Campylobacter infectivity. Especially, the developed model was further used to evaluate the infectivities of different Campylobacter species and Campylobacter isolates from wild fowl, which have not been documented previously.

This study also demonstrated successful infection and persistence ( $\leq 21$ days post-infection) (data not shown) of Campylobacter spp. from three different groups (laboratorymaintained isolates, clinical isolates from human specimens, and environmental isolates from fresh fecal samples of California gull) in newly hatched chicks. This approach also provided the sensitivity to reveal variations of Campylobacter infectivity (e.g., virulence) among the different species, isolates, and environmental genotypes evaluated. For example, the laboratory 

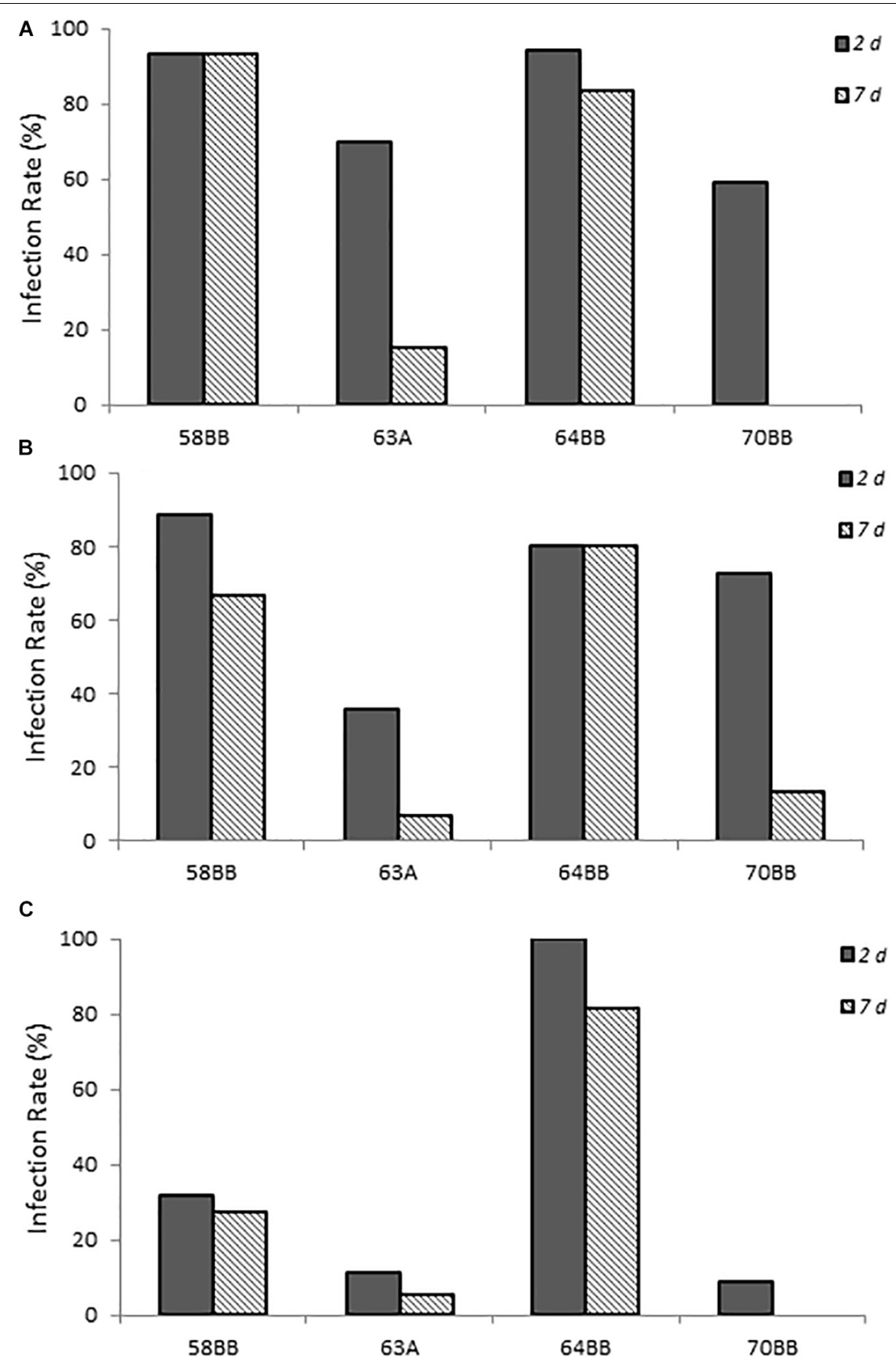

FIGURE 4 | Infection rates of environmental isolates of Campylobacter spp. (A-C) show chicks infected with Campylobacter spp. environmental isolates at doses A-C, respectively, as described in Supplementary Table S1. Percent infected was determined by dividing the number of infected chicks by the total number of chicks inoculated with the various Campylobacter species. Chicks were considered infected if fecal samples collected at the various times were positive for Campylobacter spp. as determined by using the passive-filter plating as described in section "Materials and Methods."

isolates (C. lari and C. jujuni), which had been maintained under in vitro laboratory growth conditions for an unknown extended period of time, resulted in the highest infection rates and fecal bacterial burdens, whereas $C$. coli resulted in the lowest (Figures 1, 2). More importantly, the results from the California gull isolates revealed that environmental isolates released from avian reservoirs can infect chicks. Among the gull isolates, 64BB (C. lari-like genotype) exhibited infectivity and persistence rates comparable to those of the $C$. jejuni clinical isolate. Isolate 58BB (C. lari-like genotype) exhibited infectivity and persistence rates comparable to those of the $C$. lari laboratory isolate (Figures 4, 5). The observed differences in infectivity between 


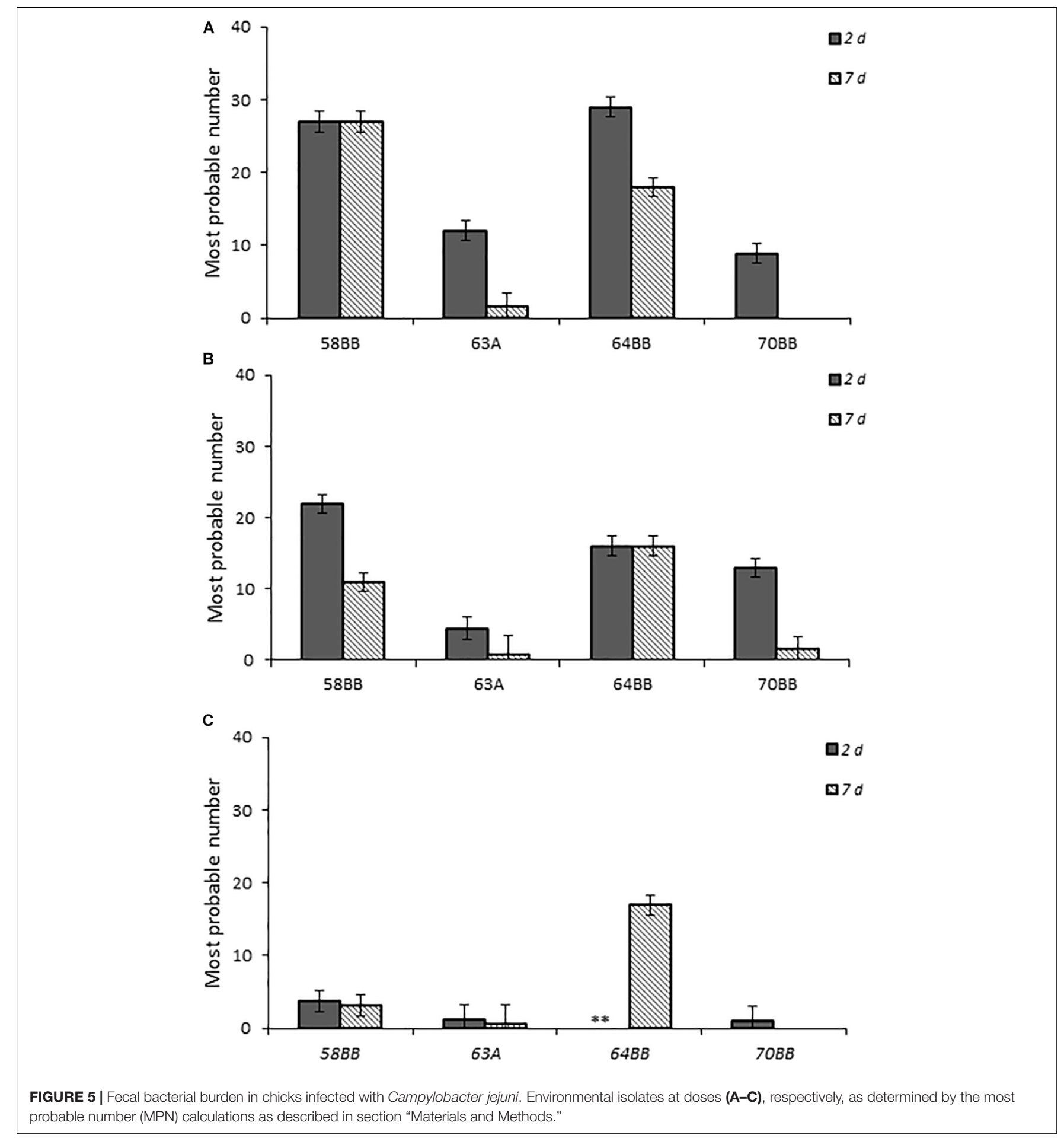

the environmental, clinical, and laboratory isolates could be attributed to differential expression of bacterial virulence factors and/or host immune responses during infection.

Furthermore, this model may help us to understand the bacterial burden and its release through defecation. Previously, it has been shown that 1-day-old chicks, orally challenged with a $10^{4} \mathrm{CFU}$ C. jejuni isolate or as few as 30 CFUs, which experienced a single passage of model chicks, achieved maximal cecal colonization within 3 days at levels of $\leq 1 \times 10^{10} \mathrm{CFU} \mathrm{g}^{-1}$ cecal contents (Cawthraw et al., 1996). In the other studies for challenge of 2-day-old chicks, $C$. jejuni has been observed at up to $\sim 10^{8}$ CFU $\mathrm{g}^{-1}$ of cecal contents (Wassenaar et al., 1993; Cawthraw et al., 1996). Once Campylobacter levels are established, they tend to remain at high levels throughout 
the life of the chick (Stern, 2008). In this study, a high bacterial cecal burden was stable at $10^{6}$ to $10^{8} \mathrm{CFU} \mathrm{\textrm {g } ^ { - 1 }}$ of cecal material throughout the duration of this experiment. The majority (57\%)of positive samples from all isolates contained $\geq 7.5 \times 10^{4} \mathrm{CFU} \mathrm{g}{ }^{-1}$ of feces. Assuming an average release of $1.5 \mathrm{~g}$ of fecal material for each defecation, $29.94 \mathrm{~g}$ of total solids per hen-day, and voids 20 times a day (Huttly et al., 1998), each individual host is capable of releasing $>3.0 \times 10^{5}$ culturable bacteria into the environment with each defecation event or $6.0 \times 10^{6}$ culturable bacteria into the environment per day.

In conclusion, this new method provides not only the ability to monitor infection through time course but also to assay virulence and other pathogen factors with relative ease. Although the relationship between the colonization in the chick ceca and the mammalian gut is unknown, the chick model still provides a relevant and natural host for Campylobacter infection. This new approach can also lend itself to identifying novel Campylobacter virulence factors, understanding host immune responses following infection with Campylobacter, screening for potential therapeutic agents, and developing vaccines relevant to the poultry industry and human health (Nedrud, 1999; Nassar, 2018). Lastly, the levels and persistence of infectious bacteria released in feces within the chick gastrointestinal tract monitored over time are important parameters when assessing the importance of waterfowl in transmission and exposure to zoonotic Campylobacter spp. in beach sites used for human recreation.

\section{DATA AVAILABILITY STATEMENT}

The datasets generated for this study are available on request to the corresponding author.

\section{ETHICS STATEMENT}

The animal study was reviewed and approved by Institutional Animal Care and Use Committee (IACUC). Written informed

\section{REFERENCES}

Babakhani, F. K., Bradley, G. A., and Joens, L. A. (1993). Newborn piglet model for campylobacteriosis. Infect. Immun. 61, 3466-3475.

Bingham-Ramos, L. K., and Hendrixson, D. R. (2008). Characterization of two putative cytochrome $\mathrm{c}$ peroxidases of Campylobacter jejuni involved in promoting commensal colonization of poultry. Infect. Immun. 76, 1105-1114. doi: 10.1128/iai.01430-07

Cawthraw, S., Wassenaar, T., Ayling, R., and Newell, D. (1996). Increased colonization potential of Campylobacter jejuni strain 81116 after passage through chickens and its implication on the rate of transmission within flocks. Epidemiol. Infect. 117, 213-215. doi: 10.1017/s0950268800001333

Clavijo, V., and Flórez, M. J. V. (2017). The gastrointestinal microbiome and its association with the control of pathogens in broiler chicken production: a review. Poult. Sci. 97, 1006-1021. doi: 10.3382/ps/pex359

Debruyne, L., Broman, T., Bergström, S., Olsen, B., On, S. L., and Vandamme, P. (2010). Campylobacter volucris sp. nov., isolated from black-headed gulls (Larus ridibundus). Int. J. Syst. Evol. Microbiol. 60, 1870-1875. doi: 10.1099/ijs. 0.013748-0 consent was obtained from the owners for the participation of their animals in this study.

\section{AUTHOR CONTRIBUTIONS}

DL and JL designed and supervised all experiments, did some data analysis, performed some experiments, and prepared the manuscript. IS did qPCR and summarized qPCR data. TG did some data analysis. KO helped to initiate the experiments and made a critical review. EV coordinated the experiments, meeting, and data analysis, and helped some manuscript preparation.

\section{FUNDING}

The United States Environmental Protection Agency (USEPA), through its Office of Research and Development, funded and managed the research described here (SSWR3.02A).

\section{ACKNOWLEDGMENTS}

We would like to thank Sharon Detmer, Diana Miller, Katrina Pratt, and MPF Federal for Animal Husbandry and to Rich Haugland for technical review of the manuscript. The study has been subjected to the USEPA's administrative review and was approved for publication. Mention of trade names or commercial products does not constitute endorsement or recommendation by the USEPA for use. The findings and conclusions in this manuscript are those of the authors and do not necessarily represent the views of the USEPA.

\section{SUPPLEMENTARY MATERIAL}

The Supplementary Material for this article can be found online at: https://www.frontiersin.org/articles/10.3389/fmicb. 2019.02292/full\#supplementary-material

Field, L. H., Underwood, J. L., Pope, L. M., and Berry, L. (1981). Intestinal colonization of neonatal animals by Campylobacter fetus subsp. jejuni. Infect. Immun. 33, 884-892.

Giallourou, N., Medlock, G. L., Bolick, D. T., Medeiros, P. H., Ledwaba, S. E., Kolling, G. L., et al. (2018). A novel mouse model of Campylobacter jejuni enteropathy and diarrhea. PLoS Pathog. 14:e1007083. doi: 10.1371/journal.ppat. 1007083

Han, S., Flamm, R., Hachem, C., Kim, H., Clarridge, J., Evans, D., et al. (1995). Transport and storage of Helicobacter pylori from gastric mucosal biopsies and clinical isolates. Eur. J. Clin. Microbiol. Infect. Dis. 14, 349-352. doi: 10.1007/ bf02116531

Hartley-Tassell, L., Day, C., Semchenko, E., Tram, G., Calderon-Gomez, L., Klipic, Z., et al. (2018). A peculiar case of Campylobacter jejuni attenuated aspartate chemosensory mutant, able to cause pathology and inflammation in avian and murine model animals. Sci. Rep. 8:12594. doi: 10.1038/s41598-018-30 604-5

Hendrixson, D. R., and DiRita, V. J. (2004). Identification of Campylobacter jejuni genes involved in commensal colonization of the chick gastrointestinal tract. Mol. Microbiol. 52, 471-484. doi: 10.1111/j.1365-2958.2004.03988.x 
Hodgson, A., McBride, B., Hudson, M., Hall, G., and Leach, S. (1998). Experimental Campylobacter infection and diarrhoea in immunodeficient mice. J. Med. Microbiol. 47, 799-809. doi: 10.1099/00222615-47-9-799

Hsieh, Y.-H., Simpson, S., Kerdahi, K., and Sulaiman, I. M. (2018). A comparative evaluation study of growth conditions for culturing the isolates of Campylobacter spp. Curr. Microbiol. 75, 71-78. doi: 10.1007/s00284-017-1 351-6

Huttly, S. R., Lanata, C. F., Yeager, B. A., Fukumoto, M., Del Aguila, R., and Kendall, C. (1998). Feces, flies, and fetor: findings from a Peruvian shantytown. Rev. Panam. Salud Pública 4, 75-79. doi: 10.1590/s1020-49891998000800001

Jarvis, B., Wilrich, C., and Wilrich, P. T. (2010). Reconsideration of the derivation of most probable numbers, their standard deviations, confidence bounds and rarity values. J. Appl. Microbiol. 109, 1660-1667. doi: 10.1111/j.1365-2672.2010. 04792.x

Jokinen, C. C., Koot, J. M., Carrillo, C. D., Gannon, V. P., Jardine, C. M., Mutschall, S. K., et al. (2012). An enhanced technique combining pre-enrichment and passive filtration increases the isolation efficiency of Campylobacter jejuni and Campylobacter coli from water and animal fecal samples. J. Microbiol. Methods 91, 506-513. doi: 10.1016/j.mimet.2012.09.005

Kapperud, G., and Rosef, O. (1983). Avian wildlife reservoir of Campylobacter fetus subsp. jejuni, yersinia spp., and salmonella spp. in Norway. Appl. Environ. Microbiol. 45, 375-380.

Lévesque, B. T., Brousseau, P., Bernier, F., Dewailly, É, and Joly, J. (2000). Study of the bacterial content of ring-billed gull droppings in relation to recreational water quality. Water Res. 34, 1089-1096. doi: 10.1016/s0043-1354(99)00266-3

Lu, J., Ryu, H., Santo Domingo, J. W., Griffith, J. F., and Ashbolt, N. (2011). Molecular detection of Campylobacter spp. in California gull (Larus californicus) excreta. Appl. Environ. Microbiol. 77, 5034-5039. doi: 10.1128/ AEM.00018-11

Lu, J., Ryu, H., Vogel, J., Santo Domingo, J., and Ashbolt, N. J. (2013). Molecular detection of Campylobacter and fecal indicators during the northern migration of sandhill cranes (Grus canadensis) at the central platte river. Appl. Environ. Microbiol. 79, 3762-37629.

Manes-Lazaro, R., Van Diemen, P., Pin, C., Mayer, M., Stevens, M., and Narbad, A. (2017). Administration of Lactobacillus johnsonii FI9785 to chickens affects colonisation by Campylobacter jejuni and the intestinal microbiota. Br. Poult. Sci. 58, 373-381. doi: 10.1080/00071668.2017.1307322

Müller, J., Schulze, F., Müller, W., and Hänel, I. (2006). PCR detection of virulenceassociated genes in Campylobacter jejuni strains with differential ability to invade Caco-2 cells and to colonize the chick gut. Vet. Microbiol. 113, 123-129. doi: 10.1016/j.vetmic.2005.10.029

Nassar, F. S. (2018). Poultry as an experimental animal model in medical research and pharmaceutical industry. Biomed. J. 2:4.

Nedrud, J. G. (1999). Animal models for gastric Helicobacter immunology and vaccine studies. Pathog. Dis. 24, 243-250. doi: 10.1111/j.1574-695x.1999. tb01290.x

Newell, D. (2001). Animal models of Campylobacter jejuni colonization and disease and the lessons to be learned from similar Helicobacter pylori models. J. Appl. Microbiol. 90, 57S-67S. doi: 10.1046/j.1365-2672.2001.01354.x

Nielsen, H. L., Ejlertsen, T., Engberg, J., and Nielsen, H. (2013). High incidence of Campylobacter concisus in gastroenteritis in North Jutland, Denmark: a population-based study. Clin. Microbiol. Infect. 19, 445-450. doi: 10.1111/j. 1469-0691.2012.03852.x

Obiri-Danso, K., Paul, N., and Jones, K. (2001). The effects of UVB and temperature on the survival of natural populations and pure cultures of Campylobacter jejuni, Camp. coli, Camp. lari and urease-positive thermophilic Campylobacters (UPTC) in surface waters. J. Appl. Microbiol. 90, 256-267. doi: 10.1046/j.1365-2672.2001.01239.x

Pitkänen, T. (2013). Review of Campylobacter spp. in drinking and environmental waters. J. Microbiol. Methods 95, 39-47. doi: 10.1016/j.mimet.2013.06.008
Quessy, S., and Messier, S. (1992). Prevalence of Salmonella spp., Campylobacter spp. and Listeria spp. in ring-billed gulls (Larus delawarensis). J. Wildlife Dis. 28, 526-531. doi: 10.7589/0090-3558-28.4.526

Ramos, R., Cerdà-Cuéllar, M., Ramírez, F., Jover, L., and Ruiz, X. (2010). Influence of refuse sites on the prevalence of Campylobacter spp. and Salmonella serovars in seagulls. Appl. Environ. Microbiol. 76, 3052-3056. doi: 10.1128/AEM. 02524-09

Ringoir, D. D., Szylo, D., and Korolik, V. (2007). Comparison of 2-day-old and 14day-old chicken colonization models for Campylobacter jejuni. FEMS Immunol. Med. Microbiol. 49, 155-158. doi: 10.1111/j.1574-695x.2006.00181.x

Salaheen, S., Tabashsum, Z., Gaspard, S., Dattilio, A., Tran, T. H., and Biswas, D. (2018). Reduced Campylobacter jejuni colonization in poultry gut with bioactive phenolics. Food Control 84, 1-7. doi: 10.1016/j.foodcont.2017. 07.021

Savill, M., Hudson, J., Ball, A., Klena, J., Scholes, P., Whyte, R., et al. (2001). Enumeration of Campylobacter in New Zealand recreational and drinking waters. J. Appl. Microbiol. 91, 38-46. doi: 10.1046/j.1365-2672.2001.01337.x

Stahl, M., and Vallance, B. A. (2015). Insights into Campylobacter jejuni colonization of the mammalian intestinal tract using a novel mouse model of infection. Gut Microb. 6, 143-148. doi: 10.1080/19490976.2015.1016691

Steele, T. W., and McDermott, S. (1984). The use of membrane filters applied directly to the surface of agar plates for the isolation of Campylobacter jejuni from feces. Pathology 16, 263-265. doi: 10.3109/00313028409068535

Stern, N. (2008). Salmonella species and Campylobacter jejuni cecal colonization model in broilers. Poult. Sci. 87, 2399-2403. doi: 10.3382/ps.2008-00140

Stoddard, R. A., Gulland, F. M., Atwill, E. R., Lawrence, J., Jang, S., and Conrad, P. A. (2005). Salmonella and Campylobacter spp. in northern elephant seals, California. Emerg. Infect. Dis. 11, 1967-1969.

Sweeney, T., Meredith, H., Vigors, S., McDonnell, M. J., Ryan, M., Thornton, K., et al. (2017). Extracts of laminarin and laminarin/fucoidan from the marine macroalgal species Laminaria digitata improved growth rate and intestinal structure in young chicks, but does not influence Campylobacter jejuni colonisation. Anim. Feed Sci. Technol. 232, 71-79. doi: 10.1016/j.anifeedsci. 2017.08.001

Wassenaar, T. M., van der Zeijst, B. A., Ayling, R., and Newell, D. G. (1993). Colonization of chicks by motility mutants of Campylobacter jejuni demonstrates the importance of flagellin A expression. Microbiology 139, 11711175. doi: 10.1099/00221287-139-6-1171

Weis, A. M., Miller, W. A., Byrne, B. A., Chouicha, N., Boyce, W. M., and Townsend, A. K. (2014). Prevalence and pathogenic potential of Campylobacter isolates from free-living, human-commensal American crows. Appl. Environ. Microbiol. Mar 1, 1639-1644. doi: 10.1128/AEM.03393-13

Weis, A. M., Storey, D. B., Taff, C. C., Townsend, A. K., Huang, B. C., Kong, N. T., et al. (2016). Genomic comparison of Campylobacter spp. and their potential for zoonotic transmission between birds, primates, and livestock. Appl. Environ. Microbiol. 15, 7165-7175. doi: 10.1128/aem.01746-16

Conflict of Interest: IS was employed by Pegasus Technical Services, Inc.

The remaining authors declare that the research was conducted in the absence of any commercial or financial relationships that could be construed as a potential conflict of interest.

Copyright (c) 2019 Lye, Struewing, Gruber, Oshima, Villegas and Lu. This is an open-access article distributed under the terms of the Creative Commons Attribution License (CC BY). The use, distribution or reproduction in other forums is permitted, provided the original author(s) and the copyright owner(s) are credited and that the original publication in this journal is cited, in accordance with accepted academic practice. No use, distribution or reproduction is permitted which does not comply with these terms. 